\title{
Best Practise aus der Lebensmittelwirtschaft
}

\section{Die geteilte Verantwortung von Lebensmittelunternehmen für nachhaltigen Konsum ist Gegenstand dieser qualitativen Untersuchung, die im Rahmen des Forschungs- projektes Geteilte Verantwortung für nachhaltigen Konsum aus Unternehmersicht - Eine internationale Studie im Lebensmittelbereich durchgeführt wurde.}

$\mathrm{D}$ Von Jasmin Pobisch ie Ergebnisse der vorliegenden Studie wurden durch eine systematische Inhaltsanalyse von schriftlichen Dokumenten wie Leitbildern, Umwelt-/Nachhaltigkeitsberichten, Geschäftsberichten und Websites ermittelt. Die Auswertung der Dokumente erfolgte durch eine systematische Inhaltsanalyse.

Die Ergebnisse der systematischen Inhaltsanalyse zeigen, dass einige deutsche Lebensmittelunternehmen ihre geteilte Verantwortung wahrnehmen, indem sie nachhaltige Produkte anbieten, Transparenz schaffen und den Dialog mit dem Konsumenten suchen, sowie zur Veränderung der institutionellen Rahmenbedingungen beitragen (1). Im Mittelpunkt der Forschung standen Unternehmen der Lebensmittelindustrie, die Mitglied der Assoziation Ökologischer Lebensmittelhersteller e.V. (AoeL) sind. Die international offene Assoziation hat rund 30 kleine bis mittelständische Mitglieder. Ziel der AoeL ist es, nachhaltigen Konsum und Verbraucherschutz im Rahmen gemeinsamer Strategien zur Entwicklung des Marktes zu fördern. Die Auswahl dieser Unternehmen ist darin begründet, dass die Mitglieder der AoeL ihre Verantwortung für nachhaltigen Konsum bereits wahrnehmen.

Als Gegenstand der Untersuchung wurden Dokumente von Unternehmen der Subbranchen Fleisch, Milch, Backwaren inklusive Dauerbackwaren, Süßwaren, Frozen-Food, Gewürze und Saucen, Tee und Kaffee und Baby-Food ausgewählt. Insgesamt haben die ausgewählten Segmente einen Anteil von über 70 Prozent am gesamten Lebensmittelmarkt. Zusätzlich wurde aus der Getränkeindustrie die Bierbranche aufgrund ihrer großen wirtschaftlichen Bedeutung ausgewählt.

Ausgehend von den Forschungsfragen und dem theoretischen Hintergrund wurde eine erste Kategorisierung basierend auf den vier Ebenen des
Nachhaltigkeits-Marketing vorgenommen: normativ, strategisch, operativ und transformativ (2). Folgendes Schema wurde systematisch durch mehrmaligen Materialdurchgang und mehrmalige Revision herausgearbeitet:

Hierbei wurden den drei Hauptkategorien jeweils Unterkategorien zugeordnet, die es ermöglichten die Textteile genau zuzuordnen. Der Prozess der Kodierung ist bereits Teil der Analyse und Interpretation des empirischen Materials.

\section{Transformation Beschaffungskette}

Nachhaltige Produktion und nachhaltige Produkte schaffen die Grundlage für nachhaltigen Konsum, indem sie dem Verbraucher überhaupt die Möglichkeit für nachhaltiges Konsumentenverhalten bieten. Im Unterschied zu konventionellen Produzenten beziehen die hier betrachteten Unternehmen ihre Rohstoffe zum Großteil vom ökologischen Landbau. Neben strategischen Überlegungen ist die Herstellung sozial-ökologischer Produkte häufig mit einer tiefen moralischen Überzeugung verbunden (3).

Die ausgewählten Unternehmen haben zu unterschiedlichen Zeitpunkten in unterschiedlichen Ausmaßen ihre Beschaffung auf den ökologischen Landbau umgestellt. Hipp (Babynahrung) ist diesbezüglich als Pioneer anzusehen, da die Umstellung dort bereits in den 50er Jahren begann. Die Mehrheit der Unternehmen wie die Hofpfisterei (Backwaren), Andechser Molkerei (Käse/Milch- produkte), Neumarkter Lammsbräu (Bier) haben ihr Umweltmanagement und eine nachhaltige Beschaffung in den 80er und 90er Jahren eingeführt, was den Paradigmenwechsel in der Gesellschaft zu dieser Zeit widerspiegelt (4). Inwieweit das Sortiment umgestellt wird, hängt allerdings nicht nur von Fragen der strategischen Positionierung ab, sondern auch von der Verfügbarkeit des entsprechenden Rohmaterials. In solchen Fällen ist es nicht nur eine Frage der umwelffreundlichen Beschaffung, sondern auch eine Frage der Transformation von Zulieferernetzwerken. So ist Salus (Kräuter, Arzneipflanzen) bis heute nicht in der Lage, alle Arzneipflanzen aus ökologischem Anbau zu beziehen, obwohl bereits 1940 die ersten ökologischen Arzneipflanzen für Salus angebaut wurden. Daher baut das Verhältnis zu den landwirtschaftlichen Zulieferbetrieben auf langfristige, co-operative Partnerschaften, die von Fairness und Vertrauen geprägt sind. Es finden Schulungen und Aufklärungsarbeiten statt, die die Landwirte in ihrer Umstellung zum ökologischen Landbau unterstïtzen sollen. In Entwicklungsländern wird dies häufig mit einem breiten sozialen Engagement verbunden.

\section{Transformation Wertschöpfungskette}

Die Transformation der Wertschöpfungskette in Richtung Nachhaltigkeit betrifft nicht nur die Angebotsseite, sondern auch die Nachfrageseite. Die meisten der analysierten Unternehmen versuchen entlang des gesamten Lebenszyklus ihre Produkte nach ökologischen und sozialen Kriterien zu optimieren. Um dies zu erreichen, werden Umweltmanagementsysteme wie ISO 14001 und EMAS eingeführt. Darüber hinaus finden kontinuierliche interne und externe Kontrollen und Mitarbeiterschulungen statt. Die Mitarbeiter der Andechser Molkerei werden beispielsweise zum Umgang mit Energie, Wasser und Reinigungsmitteln geschult. Einige der Unternehmen verwenden bereits erneuerbare Energien oder

\begin{tabular}{|l|l|l|}
\hline \multicolumn{2}{|l|}{ Tabelle 1: Kategoriensystem } \\
\hline Hauptkategorie & Unterkategorie & Kodierung \\
Angebot von Nachhaltigkeits-Produkten & Beschaffung & 1.1 \\
& Produktion & 1.2 \\
& (Retro-) Distribution & 1.3 \\
\hline \multirow{2}{*}{ Beeinflussung von Konsumentenverhalten } & Information/Transparenz & 2.1 \\
& Dialog & 2.2 \\
\hline \multirow{2}{*}{ Transformation der institutionellen } & Lokale Ebene & 3.1 \\
Rahmen-bedingungen & Nationale Ebene & 3.2 \\
& Internationale Ebene & 3.3 \\
\hline
\end{tabular}




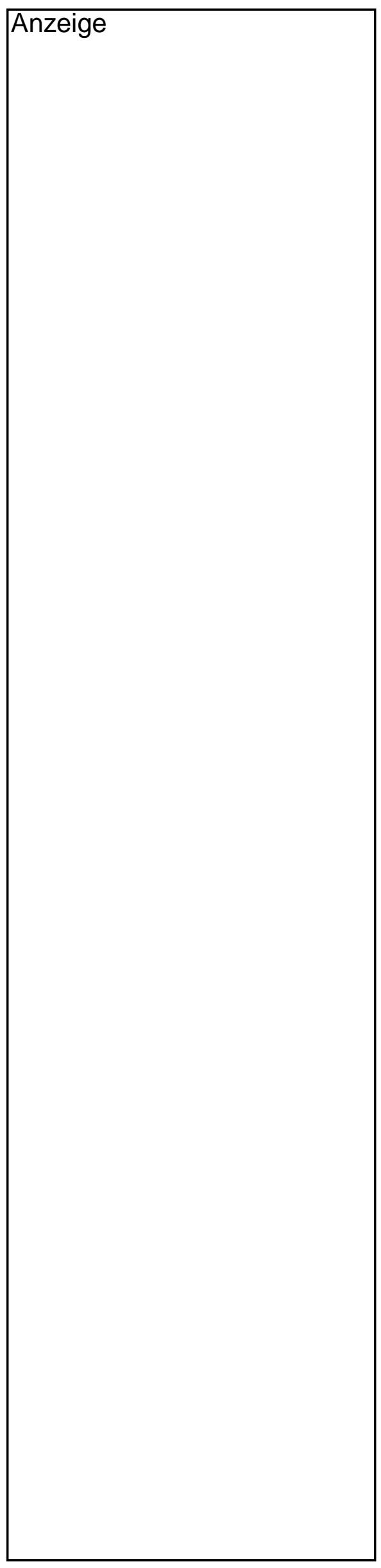

stellen ihren Fuhrpark auf den Betrieb mit Pflanzenöl um.

Parallel dazu soll der gesamte Produktlebenszyklus den Konsumenten transparenter gemacht werden, indem Firmen beispielsweise über die Herkunft ihrer Produkte, die Anbauweise und die Inhaltsstoffe aufklären. Dabei werden Informationsasymmetrien zwischen Herstellern und Konsumenten abgebaut. Ein gutes Beispiel hierfür ist die Frosta AG, die im Jahr 2003 das ,Frosta Reinheitsgebot" ins Leben gerufen hat und seitdem alle relevanten Informationen zu den Produkten auf der Verpackung abdruckt.

Umwelt- und Sozialeigenschaften eines Produktes werden oft als Vertrauenseigenschaften wahrgenommen. Im Unterschied zu Such- und Erfahrungseigenschaften können Vertrauenseigenschaften nicht vor dem Kauf erfahren werden. Aus diesem Grund sind glaubwürdige, unabhängige Labels wie Bioland, Demeter und Naturland in diesem Segment bedeutend.

\section{- Transformation Rahmenbedin- gungen}

Die aufgeführten ersten zwei Ebenen der Transformation waren stark auf den Markt gerichtet. Die dritte und letzte Ebene der Transformation bezieht sich auf nicht-marktliche Beziehungen und Interaktionen.

Durch die Mitgliedschaft und das Engagement bei der AoeL zeigen die untersuchten Unternehmen bereits ein Interesse an co-operativem Handeln auf einer sub-politischen Ebene. Viele der Mitglieder engagieren sich auch darüber hinaus. So sind die zwei Brauereien Neumarkter Lammsbräu und Riedenburger im „Lokale Agenda 21 Prozess“ beteiligt. Sie können als ,good corporate citizens" bezeichnet werden. Ein solches lokales Engagement kann auch Veränderungen auf internationaler Ebene herbeiführen. So wurden die Ergebnisse aus der Nürnberger COUP 21 Pilotstudie mit dem um Nachhaltigkeitsaspekte erweiterten N-EFQM- Modell mittlerweile zusammen mit weiteren Schlüsselthemen, wie Corporate Social Responsibility, Risikomanagement und Marketing in das neue, in Brüssel offiziell verabschiedete EFQM-Modell 2004 eingearbeitet. Auf internationaler Ebene tätig ist beispielsweise die Ulrich Walter GmbH. Sie ist Mitglied der International Federation for Organic Agriculture Movements (IFOAM), dem Bundesverband Naturkost Naturwaren Herstellung und Handel und ist Mitbegründer der International Association of Partnership (IAP). Die IAP umfasst ökologische Her- steller und Handelsunternehmen und verfolgt das Ziel, die ökologische Landbaubewegung unter einer nachhaltigen und ganzheitlichen Sicht auszuweiten. Sie kooperiert mit der Sekem-Initiative.

\section{Ausblick}

Derzeit wird die Dokumentenanalyse auf große europäische Unternehmen ausgedehnt. Dennoch sind die Grenzen der Methode deutlich erkennbar. Zum einen geben die Dokumente unter Umständen ein verzerrtes Bild der Unternehmen. Zum anderen konnten Unternehmen, die zwar interessant erschienen, aber schlecht dokumentiert waren, nicht in die Untersuchung aufgenommen werden. Um diese Grenzen zu überwinden, werden die Ergebnisse der Dokumentenanalyse mit halbstrukturierten offenen Interviews mit Unternehmensvertretern trianguliert. Darüber hinaus werden in der letzten Phase des Projektes regionale, nationale und internationale Fallstudien durchgefiuhrt.

\section{Anmerkungen}

(1) Siehe auch Belz, F-M./ J. Pobisch: Shared Responsibility for Sustainable Consumption. The Case of German Food Companies. Diskussionsbeitrag Nr. 4. Reihe Marketing und Management in der Lebensmittelbranche, München 2005.

(2) Belz, F-M.: Nachhaltigkeits-Marketing, in: Die Betriebswirtschaft (DBW). Nr. 63, 2003.

(3) Müller, S.: Normatives Nachhaltigkeits-Marketing: Motivlage von Unternehmen sozial-ökologischer Pionier- und Leadunternehmen der Lebensmittelbranche. Diskussionsbeitrag Nr. 3. Reihe Marketing und Management in der Lebensmittelbranche, München 2005.

(4) Belz, F-M.: A transition towards sustainability in the Swiss agri-food chain (1970-2000): Using and improving the multi-level perspective. In: Elzen B./ Geels F./ Green K. (eds.): System Innovation and the Transition to Sustainability. Theory, Evidence and Policy. Cheltenham 2004.

\section{Die Autorin}

Jasmin Pobisch ist wissenschaftliche Mitarbeiterin an der Professur für Betriebswirtschaftslehre Brau- und Lebensmittelindustrie der TU München.

Kontakt: TU München, Professur für BWL Brau und Lebensmittelindustrie, Alte Akademie 14, 85354 Freising-Weihenstephan. Tel. 08161-715316, E-Mail: jasmin.pobisch@wi.tum.de 
(c) 20I0 Authors; licensee IÖW and oekom verlag. This is an article distributed under the terms of the Creative Commons Attribution Non-Commercial No Derivates License (http://creativecommons.org/licenses/by-nc-nd/3.o/), which permits unrestricted use, distribution, and reproduction in any medium, provided the original work is properly cited. 